\title{
A 2-Dimensional Model for the Transmission Dynamics of Schistosomiasis in the Human-Snail Hosts
}

\author{
G. Besigye-Bafaki \\ Department of Mathematics, Makerere University, P.O. Box 7062, Kampala, Uganda
}

\begin{abstract}
The modes of transmission of schistosomiasis are considered. The transmission model is developed with an aim of establishing possible endemic equilibria within the community. Analysis of the model reveals that if the recovery rate $\beta$ of human infectives and the death rate of snails $\delta$ are increased and if at the same time the contact rate $\kappa$ of the human hosts with infested water is reduced, the disease gets eradicated from the community. Otherwise, the disease becomes endemic within the community which is a very much undesirable condition for the human population because of the debilitating nature of schistosomiasis. It is found out that this endemic state becomes stable if both the recovery rate $\beta$ of human infectives and the death rate $\delta$ of snails are reduced. It can be made unstable by de-worming the human infectives and killing snails by use of molluscicides. Educating the community about the modes of transmission of the disease helps in reducing the contact rate with infested waters and hence disease prevention.
\end{abstract}

Key words: Life cycle, life expectancy, infected human and snail populations, endemic equilibrium

\section{INTRODUCTION}

Schistosomiasis is a very severe disease because it is difficult to treat. It has a complicated life cycle which makes it more difficult to break. Within days after becoming infected, some people have a rush or itchy skin and within a month or two they may get fever, chills, cough and muscle aches. Most people have no symptoms at all in this early phase after infection. Usually the eggs of the parasite go to the liver or pass into the intestine or urinary bladder. The parasites are found within the blood vessels of the human being. Once these parasites lay eggs, the hook on them penetrates through the blood vessels and eggs are passed out through the urine or feaces with blood; a situation called haematuria. In the case of children, these worms make them lose their appetite. And as they mature, the children develop with very thin legs ${ }^{[1]}$. They do not want to play and are mentally retarded. Rarely, eggs are found in the brain or spinal cord and cause seizures, paralysis or spinal cord inflammation and thus affecting the physical and mental development of children $^{[2]}$. This is particularly true of Schistosoma mansoni infection. This debilitating nature of schistosomiasis is a markedly deterrent factor in food and other production sectors ${ }^{[1]}$. As for the older people they become weak and always complain of stomach problems. After many years of repeated infection the parasite can damage the liver, intestines, lungs or urinary bladder. This greatly diminishes the strength and production of adults. They fail to go to work which retards community growth.
This is commonly observed in rice, sugarcane growing farms and fishing villages. The situation which affects the local people in their health and socio-economic activities and government internal revenue is agriculturally and economically retarded.

Schistosomiasis is caused by parasitic flat worms of the genus Schistosoma. Male and female mate within the human host and lay eggs in the blood vessels which line the bladder and intestine. These eggs induce an immune reaction which causes the swelling of the spleen hepatosplenic and liver liver fibrosis. A portion of the eggs leave the body with the feaces or urine and find their way into the fresh water supply where they hatch into a free swimming ciliated larva called a miracidium of about $0.2 \mathrm{~mm}$ long.

If the miracidium reaches a fresh water snail of a suitable species, it penetrates and transforms into a sporocyst. The sporocyst begins an asexual phase of reproduction within the body of the snail producing thousands of daughter sporocysts. Because of this, the disease is sometimes called snail fever since the snails serve as hosts for the transmission of the disease. The infected snails then release a second form of free swimming larva called a cercaria, of about $1 \mathrm{~mm}$ long with a characteristic forked tail, into the water. The miracidia cannot infect a human being but the cercaria can. The cercaria eventually penetrate the skin of a human, loses its tail and enters the blood vessels as a Schistosomulum. It grows to adult size, matures sexually and migrates to the liver where they cause liver fluke, or migrate to the intestines or the stomach where they cause schistosomiasis.

Corresponding Author: G. Besigye-Bafaki, Department of Mathematics, Makerere University, P.O. Box 7062, Kampala, Uganda 
After the mating of the male and female schistosomes and migration to a blood vessel, egg-laying starts and the life cycle of the parasite is closed. Each pair of Schistosomulae produces hundreds to thousands of eggs per day. The eggs are spined and sieve through the tissues to the excretory $\operatorname{tract}^{[3]}$. Most of the pathology associated with infection is due to immune responses to eggs that become trapped in the host tissues.

Although research has been done on schistosomiasis in Uganda, most of the work has been mainly demographical and theoretical statistics. In this study, we are trying to demonstrate how a simple human - snail model can explain the epidemiological phenomena of schistosomiasis. The analytic model can give the consequences of the basic assumptions of the disease model and can help interpret the output of the more complex and realistic model.

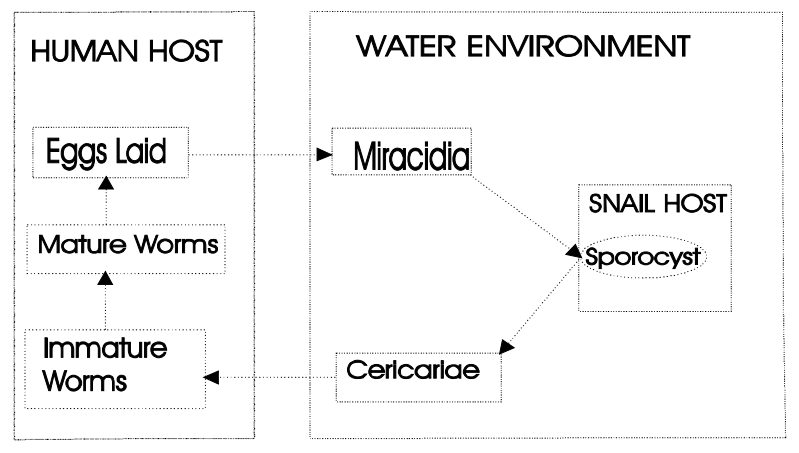

Fig. 1: A flow diagram of the life cycle of schistosoma parasites

To demonstrate how such simple analytic models can serve as useful insights to the more complex simulation models, a 2-dimensional dynamic system model of the human host and the snail host is considered. Our main concern now is to develop a mathematical model on the transmission of schistosomiasis. Before we do this, we need to consider the disease life cycle so that we are able to clearly identify the variables and the parameters of the system (Fig. 1).

Larval stage dynamics in the snail: In the case of schistosomiasis, the schistosomes do not reproduce directly within the human host. So when we consider the epidemiology of human schistosomes, we find that an individual's contribution to transmission is related quantitatively to parasite burden.

Table 1: The expected life expectancies of the human, snail hosts and other various larval stages of the parasites

\begin{tabular}{ll}
\hline Host and Parasite & Life Expectancy \\
\hline Human & $40-60$ years \\
Mature worms & $3-5$ years \\
Susceptible snail & $4-12$ weeks \\
Infected snail & $3-6$ weeks \\
Cercarial stage & $8-20$ hours \\
Miracidial stage & $4-16$ hours \\
\hline
\end{tabular}

A model of schistosome dynamics must therefore consider the intensity of infection in the human population. In contrast, schistosomes undergo extensive multiplication within the snail host. An infective snail's contribution is independent of the number of miracidia penetrating $\mathrm{it}^{[4]}$.

Table 1 gives the life expectances of the hosts and the various developmental states in the life cycle of Schistosoma mansoni ${ }^{[5]}$. Human host populations change relatively slowly and for most purposes can be assumed to be constant.

On the other hand the turnover for cercariae and miracidia populations is so rapid that larval stage densities can always be assumed to be at equilibrium for given densities of shedding snails and adult schistosomes respectively.

On the production of cercariae by infected snails, it was found out that light stimulates the shedding of cercariae. Heat is also stimulus but less effective ${ }^{[6]}$. In view of this, small pools, dams in which B.(P.) nastus productus occurs are likely to contain $S$. haematobium cercariae from mid morning until late evening ${ }^{[7]}$.

The use of such places for bathing and domestic water supplies during this period involve greater risk of infection than in the early morning. Pesigan et al. ${ }^{[8]}$ demonstrated that the mean daily output of cercariae from snails into which one miracidium had penetrated was nearly twice as high as the output from snails into which 2-5 miracidia had penetrated and suggested that this was due to crowding of sporocysts, leading to smaller development of cercariae. He further obtained evidence which supports the view that the development of sporocysts restricts the penetration of additional miracidia, although the number of parasites and the extent of their development required to produce this effect had not yet been ascertained.

\section{Dynamics for the infected human and snail populations \\ Introduction: In this model, we consider the rates of} change of the human infectives and snail infectives. Our objective is to find out conditions under which the interaction between the human hosts and water infested with cercariae will cause the disease to become established within the community. In case an endemic equilibrium exists within the community, then we should establish whether it is stable or unstable. Further analysis of the model helps us to find conditions under which we can reduce the equilibrium level leading to a possible eradication or prevention of the disease.

Variables in the model: The variables in the model are the following:

$X$ the number of infected human hosts

$Y$ the number of infected snail hosts

$N$ the human population size

$M$ the snail population size

$N-X$ the susceptible human hosts

$M-Y$ the susceptible snail hosts 
$x \quad$ the proportion of infected human hosts

$y$ the proportion of infected snail hosts

$1-x$ the proportion of susceptible human hosts

$1-y$ the proportion of susceptible snail hosts

\section{Parameters in the model}

$\alpha(Y)$ the force of infection to the susceptible human hosts and it depends on the number of infected snails, $Y$.

$\gamma(X)$ the force of infection to the susceptible snail hosts and it depends on the number of infected human hosts, $X$

$\beta \quad$ the recovery rate of the infected human hosts

$\delta \quad$ the death rate of the snail hosts

Assumptions of the model: In formulating the model, the following assumptions are made $\mathrm{e}^{[1,4,5]}$ :

The human population $\mathrm{N}$ and the snail population $\mathrm{M}$ are assumed to be constant with $\mathrm{X}$ and $\mathrm{Y}$, the infected human and snail populations respectively, as the variables.

The force of infection $\alpha(Y)$ of the human population is assumed to be an increasing concave linear function with first and second order continuous derivatives. Although the force of infection $\alpha(Y)$ depends on the infected snails $Y$, some people do not get into contact with the infested waters. This means that the force of infection is high from the start but it reduces with time as the number of the remaining susceptibles have almost no interest in entering the infested waters.

The force of infection $\gamma(X)$ of the susceptible snail hosts is assumed to be a linear function with first and second order derivatives. In this case, the more the people that are infected, the more they infest the environment and the more the snails get infected.

Snail latency is incorporated within the infective snail population meaning that those snails which are infected but are not yet infectious are counted to be in the group of infectious snails.

The model assumes that a host that recovers from the disease is immediately susceptible to reinfection (there is no immune category of hosts).

Equations and analysis of the model: According to the definitions and assumptions made in subsection 3.4, we derive the following equations for this model:

$\left.\begin{array}{rl}\frac{d X}{d t} & =\alpha(Y)(N-X)-\beta X \\ \frac{d Y}{d t} & =\gamma(X)(M-Y)-\sigma Y\end{array}\right\}$

System (1) gives the rates of change of the human and snail infectives respectively. Since $X$ and $Y$ are proportions of infected human and snail populations respectively, then $\frac{Y}{M}=y \Rightarrow Y=y M$ and $\frac{X}{N}=x \Rightarrow X=x N$ and system (1) becomes

$\left.\begin{array}{l}\frac{d x}{d t}=\alpha(y M)(1-x)-\beta x \\ \frac{d y}{d t}=\gamma(x N)(1-y)-\sigma y\end{array}\right\}$

where $\left\{\begin{array}{l}\alpha(Y)=\alpha(M y) \\ \gamma(X)=\gamma(N x)\end{array}\right.$

From assumption 2, $\alpha(Y)$ is an increasing concave function given by

$\alpha(y M)=\frac{a M y}{\beta+M y}, a>0$

Although this force of infection depends on the proportion of infected snails $y$ the relationship is not linear. From the study of the disease, even a single infected snail is able to release numerous numbers of cercariae that are able to infest the water environment ${ }^{[4]}$. Also, from assumption 3, the force of infection $\gamma(X)$ on the susceptible snails, increases as the proportion of infected human beings increases such that

$\gamma(N x)=k N x$

where $k$ is a constant.

We now find the $\mathrm{x}$-isocline and the $\mathrm{y}$-isocline by setting

$\frac{d x}{d t}=0$ and $\frac{d y}{d t}=0$

Then we get the following functions:

$f(x, y)=\alpha(y M)(1-x)-\beta x=0\}$

$g(x, y)=\gamma(x N)(1-y)-\delta y=0\}$

We now derive the $\mathrm{x}$-isocline and the $\mathrm{y}$-isocline in the $x-y$ phase plane from equations (6). Using equations (3) and (4) respectively and substituting for $\alpha(y M)$ and $\gamma(x N)$ in (6), we get

$x=\frac{a M y}{\beta^{2}+(a+\beta) M y}$

$y=\frac{k N x}{\delta+k N x}$

These are concave functions which can be represented by

$\psi(y)=\frac{a M y}{\beta^{2}+(a+\beta) M y}$

and

$\phi(x)=\frac{k N x}{\delta+k N x}$

respectively. The two isoclines intersect within the unit square as indicated in Fig. 2. 


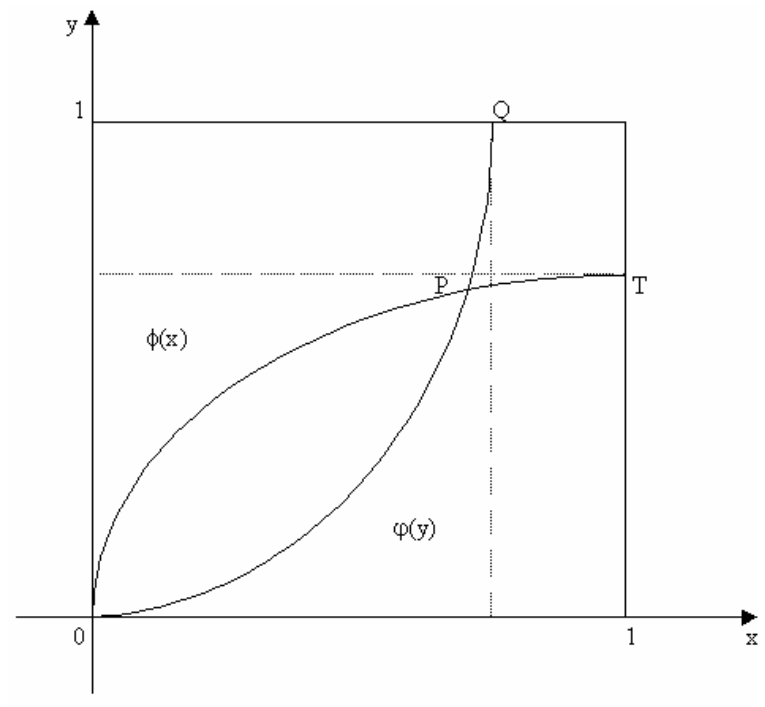

Fig. 2: The $\mathrm{x}$ and $\mathrm{y}$-isoclines intersect at the point $P\left(x^{*}, y^{*}\right)$ within the unit square

An endemic equilibrium point $P\left(x^{*}, y^{*}\right)$ exists inside the unit square $R[0,1] \times[1,0]$ as shown in Fig. 2 . The points $\mathrm{Q}$ and $\mathrm{T}$ are unstable. Any trajectories that start near $\mathrm{T}$ or $\mathrm{Q}$ move towards $\mathrm{P}$. This means that points $\mathrm{Q}$ and $\mathrm{T}$ are not easily realised and are therefore unstable and are not worth discussing.

\section{Stability of the points $(0,0)$ and $P\left(x^{*}, y^{*}\right)$}

Introduction: We need to investigate whether the points $(0,0)$ and $P\left(x^{*}, y^{*}\right)$ are stable or unstable. If the point $P\left(x^{*}, y^{*}\right)$ is stable then an endemic equilibrium exists within the community and the disease becomes chronic within the community. Such a situation is very much undesirable because of the debilitating nature of schistosomiasis. So if such a case arises, we need to find conditions under which $P\left(x^{*}, y^{*}\right)$ becomes unstable. Let us first consider any point of intersection $P(x, y)$ within the phase plane. Considering the differential equations (2) as functions of $x$ and $y$ as follows:

$$
\left.\begin{array}{l}
f(x, y)=\alpha(y M)(1-x)-\beta x \\
g(x, y)=\gamma(x N)(1-y)-\delta y
\end{array}\right\}
$$

Then at $(x, y)$

$$
\left.\begin{array}{l}
\frac{\partial f}{\partial x}=-\alpha(y M)-\beta(1-x) \\
\frac{\partial f}{\partial y}=(1-x) M \alpha^{\prime}(y M)
\end{array}\right\}
$$

$$
\left.\begin{array}{l}
\text { and } \\
\frac{\partial g}{\partial x}=(1-y) N \gamma^{\prime}(N x) \\
\frac{\partial f}{\partial y}=-\gamma^{\prime}(N x)-\delta
\end{array}\right\}
$$

and the variation matrix is given by

$$
V(x, y)=\left|\begin{array}{ll}
\frac{\partial f}{\partial x} & \frac{\partial f}{\partial y} \\
\frac{\partial g}{\partial x} & \frac{\partial g}{\partial y}
\end{array}\right|=\left|\begin{array}{cc}
-\alpha(y M)-\beta & (1-x) M \alpha^{\prime}(y M) \\
(1-y) N \gamma^{\prime}(x N) & -\gamma(x N)-\delta
\end{array}\right|
$$

Stability of the point $(\mathbf{0 , 0})$ : Using equations (12\}) and (13) in section 4.1 , we find that at $(0,0)$

$$
\begin{aligned}
& \left.\frac{\partial f}{\partial x}\right|_{x=0, y=0}=-\beta, \text { since } \alpha(0)=0 \text { and }\left.\frac{\partial f}{\partial y}\right|_{x=0, y=0}=M \alpha^{\prime}(0) \\
& \left.\frac{\partial g}{\partial x}\right|_{x=0, y=0}=N \gamma^{\prime}(0), \text { since } \gamma(0)=0 \text { and }\left.\frac{\partial g}{\partial y}\right|_{x=0, y=0}=-\delta
\end{aligned}
$$

Substituting in equation (14), the Jacobian matrix is then found to be

$$
J_{A}=\left|\begin{array}{ll}
\frac{\partial f}{\partial x} & \frac{\partial f}{\partial y} \\
\frac{\partial g}{\partial x} & \frac{\partial g}{\partial y}
\end{array}\right|=\left|\begin{array}{cc}
-\beta & M \alpha^{\prime}(0) \\
N \gamma^{\prime}(0) & -\delta
\end{array}\right|
$$

From the equation for $\alpha(y M)$ and $\gamma(x N)$ in (7) and (8) respectively, we find that

$$
\begin{aligned}
& \alpha^{\prime}(y M)=\frac{a \beta}{(\beta+M y)^{2}}, a>0 \Rightarrow \alpha^{\prime}(0)=\frac{a}{\beta} \\
& \gamma^{\prime}(x N)=k \Rightarrow \gamma^{\prime}(0)=k
\end{aligned}
$$

Substituting for $\alpha^{\prime}(0)$ and $\gamma^{\prime}(0)$ in the Jacobian matrix (15), we get

$J_{A}=\left|\begin{array}{cc}-\beta & M \alpha^{\prime}(0) \\ N \gamma^{\prime}(0) & -\delta\end{array}\right|=\left|\begin{array}{cc}-\beta & M \frac{a}{\beta} \\ N k & -\delta\end{array}\right|$

Trace $J_{A}=-(\beta+\delta)<0$

$\operatorname{Det}\left(J_{A}\right)=\beta \delta-\frac{a k}{\beta} M N$

Hence the point $(0,0)$ is stable if

$\beta^{2}>\frac{a k}{\delta} M N$

If $\beta^{2}<\frac{a k}{\delta} M N$, then the equilibrium point $(0,0)$ becomes unstable.

Stability of the point $\mathrm{P}\left(\mathrm{x}^{*}, \mathrm{y}^{*}\right)$ : We now investigate whether the endemic equilibrium $\mathrm{P}\left(\mathrm{x}^{*}, \mathrm{y}^{*}\right)$ in Fig. 3 is stable. In this case we again use the variation matrix in equation (14) to determine the characteristic function. We then apply the theory of stability ${ }^{[9]}$ to establish whether $\mathrm{P}\left(\mathrm{x}^{*}, \mathrm{y}^{*}\right)$ is stable or unstable.

At $\mathrm{P}\left(\mathrm{x}^{*}, \mathrm{y}^{*}\right)$,

$$
\begin{aligned}
& \left.\frac{\partial f}{\partial x}\right|_{x=x^{*}, y=y^{*}}=-\alpha\left(M y^{*}\right)-\beta \text { and } \\
& \left.\frac{\partial f}{\partial y}\right|_{x=x^{*}, y=y^{*}}=\left(1-x^{*}\right) M \alpha^{\prime}\left(M y^{*}\right) \\
& \left.\frac{\partial g}{\partial x}\right|_{x=x^{*}, y=y^{*}}=\left(1-y^{*}\right) N \gamma^{\prime}\left(N x^{*}\right) \text { and }
\end{aligned}
$$


$\left.\frac{\partial g}{\partial y}\right|_{x=x^{*}, y=y^{*}}=-\gamma\left(N x^{*}\right)-\delta$

We now consider these variations at $\mathrm{P}\left(\mathrm{x}^{*}, \mathrm{y}^{*}\right)$ and get the variation matrix given by

$V\left(x^{*}, y^{*}\right)=\left|\begin{array}{ll}\frac{\partial f}{\partial x} & \frac{\partial f}{\partial y} \\ \frac{\partial g}{\partial x} & \frac{\partial g}{\partial y}\end{array}\right|=\left|\begin{array}{cc}-\alpha\left(y^{*} M\right)-\beta & \left(1-x^{*}\right) M \alpha^{\prime}\left(y^{*} M\right) \\ \left(1-y^{*}\right) N \gamma^{\prime}\left(x^{*} N\right) & -\gamma\left(x^{*} N\right)-\delta\end{array}\right|$

since,

$\alpha\left(M y^{*}\right)\left(1-x^{*}\right)-\beta x^{*}=0$

by equation (3), we substitute for $\alpha\left(M y^{*}\right)$ in equation (23) to get

$\frac{a M y^{*}}{\beta+M y^{*}}\left(1-x^{*}\right)-\beta x^{*}=0$

Solvig for $\mathrm{x}^{*}$ we find that

$x^{*}=\frac{a M y^{*}}{\beta^{2}+(a+\beta) M y^{*}}$

Similary, using equation (4), we find that

$k N x^{*}\left(1-y^{*}\right)-\delta y^{*}=0$

Solving for $\mathrm{y}^{*}$, we get

$y^{*}=\frac{k N x^{*}}{\delta+k N x^{*}}$

Substituting for $\mathrm{y}^{*}$ in (25), we get

$x^{*}=\frac{a k M N-\beta^{2} \delta}{\beta^{2}+(a+\beta) k N M}$

and $0<x^{*}<1$ if the numerator $a k N M-\beta^{2} \delta>0$.

Using equation (28) to get the value for $x^{*}$, and substituting this value in (27), we get

$$
y^{*}=\frac{\left(a k N M-\beta^{2} \delta\right) k N}{\beta^{2} \delta+\left((a+\beta) \delta+a-\beta^{2} \delta\right) k N M}
$$

Both $\mathrm{x}^{*}$ and $\mathrm{y}^{*}$ (according to equations (28) and (29) are positive if $\beta^{2}<\frac{a k}{\delta} M N$.

Our main interest is to investigate the stability of the point $\left(x^{*}, y^{*}\right)>(0,0)$. The point $P\left(x^{*}, y^{*}\right)$ is stable if $\operatorname{tr}(V)$ (trace of $\mathrm{V}$ ) is negative $\operatorname{det}(V)$ (the determinant of the Jacobian matrix $\mathrm{V}$ in (22) is positive. From the Jacobian matrix $\mathrm{V}$ in (22),

$\operatorname{tr}(V)=-\left[\beta+\delta+\alpha(x * M)+\gamma\left(N x^{*}\right)<0\right]$

which is negative and hence the point $P\left(x^{*}, y^{*}\right)$ is an attractor as shown in Fig. 3.

In the figure, the arrows facing vertically upwards indicate that the corresponding variable $x$ or $y$ is increasing in the sub-region of the phase plane while those facing vertically downwards indicate that the corresponding variable $x$ or $y$ is decreasing in the sub-region of the phase plane. The arrows pointing at

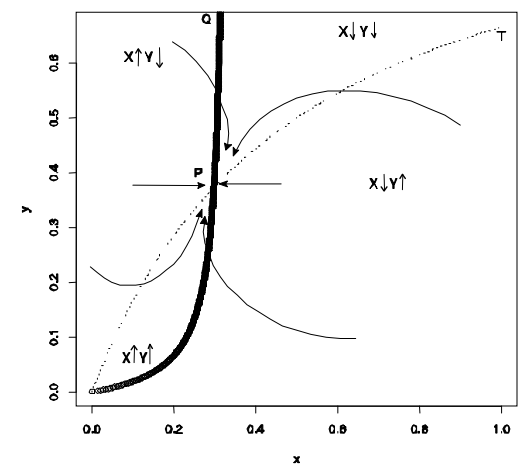

Fig. 3: Typical trajectories showing that the point $P$ is a stable endemic equilibrium

the point $P$ indicate that all trajectories move towards $P$ meaning that $P$ is an attractor.

Further more the determinant of matrix $\mathrm{V}$ is given by

$$
\begin{aligned}
& \operatorname{det}(V)=-\left[\beta+\delta+\alpha\left(x^{*} M\right)+\gamma\left(N x^{*}\right)\right]- \\
& \left(1-x^{*}\right)\left(1-y^{*}\right) \gamma^{\prime}\left(N x^{*}\right) \alpha^{\prime}\left(M y^{*}\right) N M
\end{aligned}
$$

We need to find out whether $\operatorname{det}(V)>0$ for the equilibrium point $P\left(x^{*}, y^{*}\right)$ to be stable. Rearranging equations (23) and (26) by substituting in expressions for $\mathrm{x}^{*}$ and $\mathrm{y}^{*}$ in (28) and (29) respectively, we get expressions for $\alpha\left(M y^{*}\right)$ and $\gamma\left(N x^{*}\right)$ as follows:

$\alpha\left(M y^{*}\right)=$

$\frac{\left(a k N M-\beta^{2} \delta\right) a k N M}{\beta\left[\beta^{2} \delta+\left((a+\beta) \delta+a-\beta^{2} \delta\right) k N M\right]+\left(a k N M-\beta^{2} \delta\right) k N M}$

$\gamma\left(N x^{*}\right)=\frac{\left(a k N M-\beta^{2} \delta\right)}{\beta^{2}+(a+\beta) k N M} k N$

If $\operatorname{det}(\mathrm{V})>0$, then

$\left[\beta+\alpha\left(M y^{*}\right)\right]\left[\delta+\gamma\left(N x^{*}\right)\right]>$

$\left(1-x^{*}\right)\left(1-y^{*}\right) \gamma^{\prime}\left(N x^{*}\right) \alpha^{\prime}\left(M y^{*}\right) N M$

Using equations (28) and (29), we get all other terms in inequality (34) in terms of model paramaters. Substituting them in inequality (34), we find that

$\beta^{2}<\frac{a k}{\delta} M N$

is a condition for $P\left(x^{*}, y^{*}\right)$ to be a stable equilibrium point. It is noted from the Jacobian matrix that the trace of the Jacobian matrix $\mathrm{V}$ is negative and its determinant is positive if inequality (35) is satisfied. Comparing inequalities (19) and (35), it is noted that if the point $(0,0)$ is unstable, then the disease attains a stable endemic state $P\left(x^{*}, y^{*}\right)$ within the community as shown in Fig. 3. 


\section{DISCUSSION AND CONCLUSION}

We realise from equations (32) and (33) that the force of infection, $\alpha\left(M y^{*}\right)$ on the human population and the force of infection, $\gamma\left(N x^{*}\right)$ on the snails are both positive if $\beta^{2}<\frac{a k}{\delta} M N$, which is the same condition given

by inequality (35). If inequality (35) is satisfied, the disease attains a stable endemic state $P\left(x^{*}, y^{*}\right)$ within the community and the disease becomes chronic. Although people are able to survive with the disease, such a situation is very much undesirable because of the debilitating nature of schistosomiasis. With reference to (28\}) and(29), we note that in order to reduce the proportion of infective human population at $P\left(x^{*}, y^{*}\right)$, we need to increase the recovery rate $\beta$ and also to increase the death rate of infective snails $\delta$ and reduce the contact rate $\kappa$.

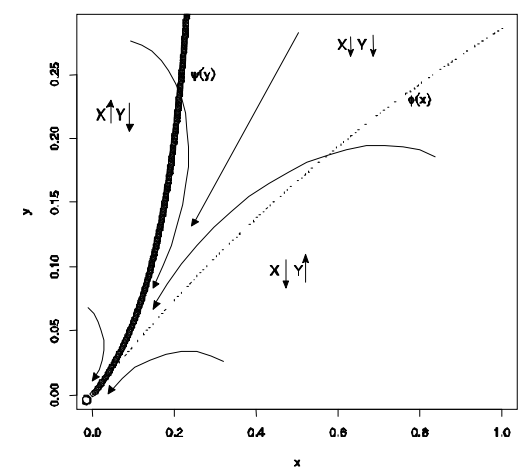

Fig. 4: Trajectories showing that the point $O(0,0)$ is a stable

Then the point $P\left(x^{*}, y^{*}\right)$ becomes closer to the origin and the disease may eventually be eradicated as shown in Fig. 4. Schistosomiasis is a focal disease which is mainly rural. It is commonly endemic in fishing villages, irrigation schemes, rice growing areas and also in sugarcane growing regions. To increase $\beta$, the public health sector needs to spot such areas, then carry out control measures e.g mass drug administration
(MDA) to infected persons in such areas. The death rate $\delta$ can also be increased by killing the snails using molluscicides. Snail eradication cannot easily be achieved, but some techniques are known to reduce snail multiplication. The contact rate $\kappa$ can also be reduced by educating the masses about the causes of schistosomiasis and the modes of transmission. Since water is necessary for life, it cannot be avoided. The only way to avoid direct water contact is to put on protective clothing like gum boots, swimming costumes and gloves before entering the water.

\section{ACKNOWLEDGEMENTS}

This work has been supported by NUFU, a Norwegian Government-Makerere University collaboration programme. More support has been offered by the Institute of Mathematical Sciences, Norwegian University for Science and Technology (NTNU), who accommodated us during the final writing of this paper. We also thank Prof. Steinar Engen and his Statistical Research members: Øyvind Bakke, Jarle Tufto and Magnar Lillegard, who read through and made positive comments and guidance on the write up of this manuscript. 


\section{REFERENCES}

1. Makanga, B., 1991. The molluscicidal properties of balanites Wilsoniana Dawe and Sprague and Khaya grandifoliola C.D.C. Ph. D. Thesis, Department of Zoology, Makerere University, Kampala.

2. Cowper, S.G., 1971. A synopsis of African Bilharziasis, London-Lewis.

3. Jordan, P. and G. Webbe, 1982. Schistosomiasis Epidemiology, Treatment and Control, Londonlewis, London.

4. Woolhouse, M.E.J., 1994. Epidemiology of Human Schistosomes: Parasitic and Infectious Diseases. Academic Press, Inc. London, pp: 197-217.

5. Anderson, R.M. and R.M. May, 1992. Infectious Diseases of Humans, Dynamics and Control. Oxford Science Publications, Oxford University Press, Inc. NewYork.
6. MacCelland, W.P.J., 1964. Schistosomiasis mansoni in the Nile delta. Ann. Trop. Med. Parasitol., 58: 265-267.

7. Kinoti, G., 1963. Schistosomiasis Infection on the coastal Areas of Tanzania, Reprint of the East African Institute of Medical Research, Govt. Printer, Nairobi.

8. Pesigan, P.T., M. Forooq and N. Hairston, 1958. Studies on Schistosoma japonicum infections in the Phillippines. Bull. World Health Organization, 18: $54-59$.

9. ELsgolts, L., 1980. Differential Equations and the Calculus of Variations. MIR Publishers, Moscow. 